\title{
CAPACIDAD INFECTIVA DE PROMASTIGOTES EN FASE ESTACIONARIA DE Leishmania (Viannia) braziliensis y Leishmania (Viannia) peruviana, EN LÍNEA CELULAR DH82
}

\author{
Karen Daphne Calvay-Sánchez,a, Nyshon Máximo Rojas-Palomino 2,a, Aidé Clorinda Sandoval-Juarez,b, \\ Alberto Cisneros-Tarmeño ${ }^{3, c}$, Cristian Obregón-Cahuayaa,d, Gloria Sonia Minaya-Gómez ${ }^{2, e}$
}

\begin{abstract}
RESUMEN
Objetivos. Determinar la capacidad infectiva de los promastigotes de Leishmania (V.) peruviana y Leishmania (V.) braziliensis en la línea celular macrófago-monocítica de Canis familiaris DH82. Materiales y métodos. Se realizó un estudio experimental durante los meses de enero a diciembre de 2013. Se utilizaron cepas referenciales de Leishmania (V.) braziliensis MHOM/PE/84/LC53 y Leishmania (V.) peruviana MHOM/PE/84/LC26. La línea celular fue infectada con promastigotes en fase estacionaria y la capacidad infectiva fue determinada como el producto del porcentaje de macrófagos infectados por el promedio de amastigotes por macrófago infectado, observado al microscopio de epifluorescencia. Resultados. El 13\% de formas metacíclicas para Leishmania (V.) braziliensis correspondió al día 17,5 posinoculación y para Leishmania (V.) peruviana un porcentaje de $9,5 \%$ en el día 14,5 . No se encontró diferencia significativa entre la capacidad infectiva de los promastigotes en fase estacionaria de ambas especies. Conclusiones. Se recomienda evaluar la capacidad infectiva de los promastigotes metacíclicos de cepas de Leishmania (V.) peruviana y Leishmania (V.) braziliensis en líneas celulares, a fin de determinar el modelo de infección in vitro más adecuado, que permita efectuar estudios de susceptibilidad a las drogas leishmanicidas de mayor eficacia para el control de la enfermedad.
\end{abstract}

Palabras clave: Leishmaniasis; Virulencia; Línea celular, Leishmania braziliensis (fuente: DeCS BIREME).

\section{INFECTIVITY OF PROMASTIGOTES IN STATIONARY PHASE OF Leishmania (Viannia) braziliensis AND Leishmania (Viannia) peruviana, IN CELL LINE DH82}

\begin{abstract}
Objectives. To determine the infectivity of promastigotes of Leishmania (V.) peruviana and Leishmania (V.) braziliensis in monocyte-macrophage cell line DH82 of Canis familiaris. Materials and methods. Was conducted a experimental study during the months of january to december 2013. Were used strains of Leishmania were used (V.) braziliensis MHOM / PE / 84 / LC53 and Leishmania (V.) peruviana MHOM / PE / 84 / LC26. The cell line was infected with stationary phase promastigotes and infectivity was determined as the product of percent infected macrophages average amastigotes per macrophage observed in epifluorescence microscope. Results. $13 \%$ of metacyclic forms to Leishmania (V.) braziliensis corresponded to 17.5 days post inoculation and Leishmania (V.) peruviana a percentage of $9.5 \%$ on the day 14.5 . No significant difference was found between infectivity of stationary phase promastigotes of both species. Conclusions. It is recommended assess the infectivity of metacyclic promastigotes peruviana strains of Leishmania (V.) and Leishmania (V.) braziliensis cell lines in order to determine the most appropriate model in vitro infection, allowing leishmanicidas make the drug more effective susceptibility studies for disease control.
\end{abstract}

Key words: Leishmaniasis; Virulence; Cell line, Leishmania braziliensis (source: MeSH NLM).

\footnotetext{
Facultad de Ciencias Biológicas. Universidad Nacional de Trujillo. Trujillo, Perú.

Laboratorio de Leishmaniosis, Centro Nacional de Salud Pública. Instituto Nacional de Salud. Lima, Perú

Laboratorio de cultivo celular, Centro Nacional de Salud Pública, Instituto Nacional de Salud. Lima, Perú.

Unidad de Análisis y Generación de Evidencias en Salud Pública. Centro Nacional de Salud Pública. Instituto Nacional de Salud. Lima, Perú.

a Biólogo microbiólogo; ${ }^{\mathrm{b}}$ biólogo; ${ }^{\mathrm{c}}$ médico veterinario; ${ }^{\mathrm{d}}$ licenciado en Estadística; ${ }^{\mathrm{e}}$ biólogo, máster en Medicina Tropical y Salud Internacional Recibido: 19-08-14 Aprobado: 04-03-15
}

Citar como: Calvay-Sánchez KD, Rojas-Palomino NM, Sandoval-Juarez AC, Cisneros-Tarmeño A, Obregón-Cahuaya C, Minaya-Gómez GS. Capacidad infectiva de promastigotes en fase estacionaria de Leishmania (Viannia) braziliensis y Leishmania (Viannia) peruviana, en línea celular DH82. Rev Peru Med Exp Salud Publica. 2015;32(1):33-40. 


\section{INTRODUCCIÓN}

Laleishmaniosis es una enfermedad metaxénica parasitaria que afecta aproximadamente a 12 millones de personas en el mundo, y que se manifiesta con formas clínicas variadas como la leishmaniosis cutánea, leishmaniosis mucosa y la leishmaniosis visceral (1). Se ha reportado en 98 países y se ha estimado un total de 0,2 a 0,4 millones de casos por año para la leishmaniosis visceral y 0,7 a 1,2 millones de casos estimados por año para las formas cutáneas de la enfermedad, siendo de Afganistán, Argelia, Irán, Etiopía, Norte de Sudan, Siria, Brasil, Colombia, Costa Rica y Perú los países que reportan entre el 70 a $75 \%$ de la incidencia global estimada para esta forma clínica (2); en el Perú se ha reportado en los últimos 5 años, más de 6 mil casos por año, lo cual representa aproximadamente el $9,6 \%$ de casos reportados en América ${ }^{(3)}$; es la segunda endemia de tipo tropical y la tercera causa de morbilidad por enfermedades transmisibles luego de la malaria y la tuberculosis ${ }^{(1)}$.

La virulencia es el grado de patogenicidad, inherente de un agente infeccioso, para causar o infringir daño a una persona bajo condiciones clínicas ${ }^{(4,5)}$, y que involucra diversos factores como los microbiológicos, patológicos y genéticos ${ }^{(5)}$, en el caso de los parásitos intracelulares como Leishmania, la virulencia está relacionada, además, con la capacidad de sobrevivencia, la diferenciación y la multiplicación en condiciones adversas del fagolisosoma de los macrófagos del hospedador vertebrado ${ }^{(6)}$, el cual contiene enzimas proteolíticas que pueden matar o digerir al parásito; si el parásito logra evadir esta respuesta, se multiplica en el macrófago, provocando su lisis y liberándose como amastigote, siendo posteriormente, fagocitados por otros macrófagos, células dendríticas y dentro de fibroblastos ${ }^{(7,8)}$, mientras que la capacidad infectiva o infectividad es un término que define a la capacidad de un microorganismo para ingresar, diseminarse y reproducirse o multiplicarse al interior de un hospedero ya sea a nivel celular, de tejido u órganos ${ }^{(9,10)}$; anteriormente esta cualidad del microrganismo fue definido como "agresividad", término que entró en desuso debido al significado amplio e inespecífico que representa ${ }^{(11,12)}$.

El género Leishmania, presenta durante su ciclo dos formas evolutivas: el estadio amastigote, forma no flagelada presente en los macrófagos del hospedero mamífero y el estadio promastigote, forma flagelada presente en el vector ${ }^{(13)}$ y que involucra a varias especies del género Lutzomyia; son las hembras hematófagas quienes mediante la picadura de un hospedero mamífero se infectan al ingerir las formas amastigotes junto con la sangre; y es en el intestino medio del vector donde los amastigotes se convierten de la forma no flagelada e inmóvil a la forma flagelada como consecuencia del cambio de condiciones de $\mathrm{pH}$ y temperatura (14); se multiplican rápidamente por división binaria y a los pocos días, inician el proceso de migración hacia el intestino medio torácico y a la válvula cardiaca, adhiriéndose a las microvellosidades del esófago y la faringe, por medio de su flagelo; en este proceso, el parásito sufre cambios genéticos, bioquímicos y morfológicos ${ }^{(14)}$. Esta migración tiene consecuencias adversas en el vector, principalmente en el proceso de ingesta de la sangre, debido al bloqueo de las válvulas estomodeal y cardiaca, además del intestino medio por la acumulación de un gel rico en carbohidratos que es formado por los productos de secreción del parásito, que permite la acumulación de un mayor número estos; debido a que estas válvulas se encuentran bloqueadas, el insecto vector debe realizar más de un intento de succión durante la picadura y cuando lo logra, se permite el flujo en ambas direcciones de la sangre, del mamífero hacia el vector y de los parásitos del vector hacia el mamífero ${ }^{(14)}$.

El proceso de metaciclogénesis es la transformación de amastigotes a promastigotes avirulentos o no infectivos (procíclicos) y de estos en promastigotes virulentos (metacíclicos); tiene lugar en el intestino medio del vector con una duración de 10 a 11 días, de los cuales requiere de 3 días para el cambio de amastigotes a promastigotes avirulentos y de 7 a 8 días para la transformación de estos a promastigotes virulentos, otras investigaciones señalan que el proceso de metaciclogénesis puede alcanzar una duración de diez a catorce días ${ }^{(13,15)}$.

Para lograr una aproximación de los procesos involucrados en la infección natural, los estudios de infección in vitro de Leishmania requieren de la obtención de los promastigotes metacíclicos, estadio que cuenta con mayor virulencia en comparación con los promastigotes procíclicos ${ }^{(16,17)}$ por la presencia de glicoproteínas, principalmente la glicoproteína 63 (gp63) (13), que es una metaloproteasa de zinc abundante en la superficie de los promastigotes. Otros estudios realizados en el subgénero Leishmania, revelan que durante la metaciclogénesis, el lipofosfoglicano (LPG), un glicolípido presente también en la superficie de los promastigotes del parásito que interactúa con la superficie epitelial del intestino medio permitiendo su adhesión, sufre modificaciones estructurales en sus cadenas laterales que involucran a monosacáridos, en el caso de Leishmania (L.) major involucra a una hexosa (galactosa) y a una pentosa (arabinosa) ${ }^{(18)}, y$ en $L$. (V.) braziliensis, se ha reportado que el LPG se encuentra en menor cantidad en comparación a las especies del subgénero Leishmania, pero al igual que en estas, las formas promastigotes cuentan con una 
mayor proporción de estas moléculas en comparación de las formas metacíclicas. Mediante pruebas inmunohistoquímicas se demostró que el LPG en las formas promastigotes procíclicas y metacíclicas que se encuentran adheridas al epitelio del intestino medio del vector, cuentan con residuos de $\beta(1,3)$ glucosa; otras modificaciones que han sido involucradas en esta interacción del parásito con el vector son la galactosa, la manosa y la glucosa ${ }^{(19,20)}$. La transformación de promastigotes procíclicos a metacíclicos involucra modificaciones bioquímicas, moleculares y genéticas que proporcionan al promastigote metacíclico resistencia a la lisis mediada por el complemento ${ }^{(21,22)}$, facilitan el proceso de fagocitosis por los macrófagos (22), aseguran la supervivencia del parásito en condiciones adversas en el fagolisosoma del macrófago, inducen la formación de proteínas de la familia de las quinasas y regulan la producción del óxido nítrico en las vacuolas fagocitóforas ${ }^{(20,23)}$.

El objetivo de esta investigación fue determinar la capacidad infectiva de los promastigotes Leishmania (V.) braziliensis y Leishmania (V.) peruviana en fase estacionaria ${ }^{(7,15,24)}$, en condiciones in vitro en la línea celular DH82; así mismo, contribuir en la búsqueda de un modelo celular con fines de infección in vitro que permita realizar ensayos de susceptibilidad en formas amastigotes a drogas leishmanicidas y la evaluación de nuevas alternativas terapéuticas de bajo costo y menor toxicidad.

\section{MATERIALES Y MÉTODOS}

Se realizó un estudio de tipo experimental en los ambientes del Laboratorio de Leishmaniosis del Centro Nacional de Salud Pública, Instituto Nacional de Salud, durante los meses de enero a diciembre de 2013.

Se utilizaron cepas referenciales de Leishmania (V.) braziliensis MHOM/PE/84/LC53 y Leishmania (V.) peruviana MHOM/PE/84/LC26. Estas cepas criopreservadas a $-80{ }^{\circ} \mathrm{C}$, fueron descongeladas y cultivadas a $24^{\circ} \mathrm{C}$ en medio Drosophila de Schneider's (Gibco-Life technologies) a pH 6,5 suplementado con $10 \%$ de suero fetal bovino inactivado (SFBI) a $56{ }^{\circ} \mathrm{C}$ y gentamicina (medio Schneider's completo pH de 7,0), conservándose por pasajes sucesivos cada 20 días.

\section{CURVA DE CRECIMIENTO}

Para obtener la curva de crecimiento, las cepas de Leishmania (V.) braziliensis y Leishmania (V.) peruviana fueron cultivadas en $2 \mathrm{~mL}$ de medio
Schneider's completo a una concentración de $5 \mathrm{x}$ $10^{2}$ parásitos $/ \mathrm{mL}$ e incubados a $29{ }^{\circ} \mathrm{C}$, etapa que correspondió al punto inicial o T0 de la curva de crecimiento. El recuento de parásitos mediante cámara hematocitométrica, se efectuó cada $12 \mathrm{~h}$, durante 18 días consecutivos.

\section{RECUENTO DE FORMAS METACÍCLICAS}

Se tomó una alícuota del cultivo de parásitos cada 12 h durante los 12 días de la fase estacionaria, se colorearon por tinción Giemsa, para determinar el día posinoculación con el mayor porcentaje de formas promastigotes metacíclicos, estas fueron definidas por la forma y proporción del cuerpo con el flagelo. Los recuentos de 100 parásitos se realizaron por duplicado.

\section{LÍNEA CELULAR}

Se utilizó la línea celular ATCC de macrófago de Canis familiaris DH82, proporcionada por el Área de Cultivo Celular del Instituto Nacional de Salud (INS), y que se encuentra criopreservada a -80 ${ }^{\circ} \mathrm{C}$; esta línea celular fue descongelada, cultivada y mantenida en medio mínimo esencial (MEM) suplementado con $15 \%$ de SFBI más solución de antibiótico-antimicótico (medio MEM completo), a $37^{\circ} \mathrm{C}$, en presencia de $\mathrm{CO}_{2}$ al $5 \%$, para el presente estudio se empleó la línea celular en fase logarítmica obtenidos a partir del cuarto pasaje.

\section{INFECCIÓN IN VITRO}

Los macrófagos para los del grupos control y de infección, obtenidos de la fase logarítmica, fueron depositados a una concentración de $4 \times 10^{4} \mathrm{cel} / \mathrm{mL}$, sobre una laminilla de vidrio colocada en la base de los 24 pocillos de placas para cultivo celular; las placas fueron incubadas a $37^{\circ} \mathrm{C}$ por $72 \mathrm{~h}$ en presencia de $\mathrm{CO}_{2}$ al $5 \%$.

Los parásitos en fase estacionaria fueron colectados del medio Schneider's del día con el mayor porcentaje de las formas promastigotes metacíclicos; se centrifugaron a $5000 \mathrm{rpm}$ por $5 \mathrm{~min}$, se lavaron en solución salina fisiológica (SSF) estéril tres veces, y fueron resuspendidos en medio MEM completo.

El ensayo de infección se realizó en dos grupos; en ambos, los parásitos fueron inoculados a 10, 15 y 20 parásitos/células aproximadamente, en el cultivo celular de macrófagos de 72 horas de incubación; las placas fueron incubadas a $37{ }^{\circ} \mathrm{C}$ con $5 \%$ de $\mathrm{CO}_{2}$. Luego de 
$24 \mathrm{~h}$ de infección, se retiró el primer grupo de laminillas de vidrio correspondiente al tiempo inicial o cero (TO) de infección; en el segundo grupo, el medio de cultivo conteniendo parásitos no internalizados en los macrófagos se retiró a las 24 h y se restituyó con medio MEM completo, y se incubó por otras $24 \mathrm{~h}$ a $37^{\circ} \mathrm{C}$, con $5 \%$ de $\mathrm{CO}_{2}$, al término de los cuales se retiraron las laminillas correspondiendo al tiempo 1 (T1) de infección; en ambos grupos las laminillas fueron retiradas agregándosele suero humano con inmunoglobulina G (lgG) anti-Leishmania, posteriormente, se adicionó anti-lgG humano conjugado con isotiocianato de fluoresceína (Sigma), las láminas fueron observadas empleando microscopio de epifluorescencia (Axio Scope.A1 - CarlZeiss).

\section{EVALUACIÓN DE LA CAPACIDAD INFECTIVA}

Se evaluaron 100 macrófagos por grupo (grupo control, grupo T0 y T1 de infección), se consideró el promedio de amastigotes por macrófago y el porcentaje de macrófagos infectados; para ello se empleó el objetivo de 100x en microscopio de epifluorescencia.

La determinación de la capacidad infectiva $(\mathrm{Cl})$, siguiendo lo reportado por Monzote et al. ${ }^{(25)}$, corresponde al producto del promedio de amastigotes por macrófagos (Ø) infectados por el porcentaje de macrófago infectado.

\section{ANÁLISIS DE DATOS}

El promedio y la desviación estándar de los amastigotes por macrófago y el porcentaje de macrófagos infectados fueron determinados en el programa Microsoft Excel 2010; el análisis estadístico se realizó inicialmente mediante la prueba no paramétrica de Kruskal-Wallis, para determinar si la diferencia entre los resultados a diferentes proporciones de parásitos/célula era significativa $(p<0,05)$ seguido por la prueba de Wilcoxon para determinar si existía diferencia significativa entre Leishmania (V.) braziliensis y Leishmania (V.) peruviana $(p<0,05)$, empleando el programa estadístico $R$ versión 3.1.2.

\section{RESULTADOS}

Las curvas de crecimiento obtenidas para Leishmania (V.) braziliensis y Leishmania (V.) peruviana, se observó, que la fase de latencia duró aproximadamente $24 \mathrm{~h}$, iniciándose la fase exponencial temprana al segundo día posinoculación, la fase exponencial se prolongó hasta el día 6; a continuación, se observó que ambas especies entraron en fase estacionaria al día 7 extendiéndose por 12 días (Figura 1).

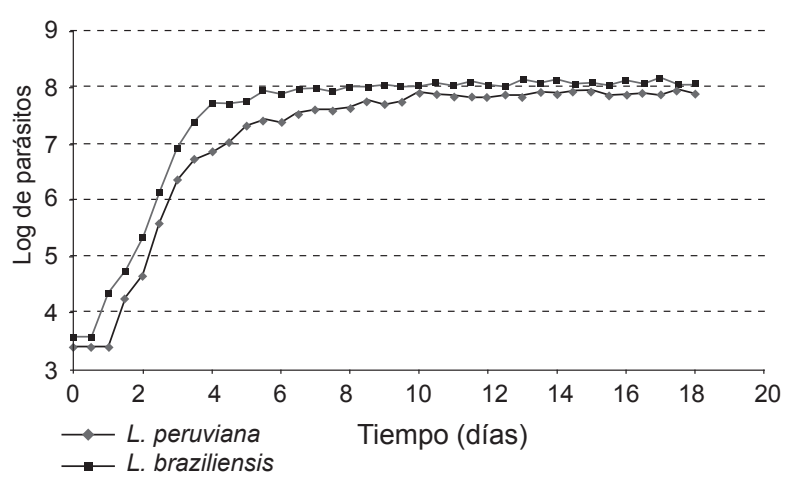

Figura 1. Curva de crecimiento de Leishmania (V.) peruviana y $L$. (V.) braziliensis en medio Schneider's a $\mathrm{pH} 7,0$, con recuentos cada $12 \mathrm{~h}$

Del recuento de parásitos de la fase estacionaria, se observó que Leishmania (V.) braziliensis alcanzó un máximo del $13 \%$ de formas metacíclicas a los 17,5 días posinoculación; y Leishmania (V.) peruviana alcanzó el 9,5\% de formas metacíclicas a los 14,5 días, los obtención de promastigotes en fase estacionaria para los ensayos de infección, se realizó a partir de los días obtenidos con el mayor porcentaje de formas promastigotes metacíclicos determinados en la presente etapa.

\section{INFECCIÓN IN VITRO}

En la infección de los macrófagos de perro $\mathrm{DH} 82$ con los promastigotes en fase estacionaria, la capacidad infectiva inicial $\left(T_{0}\right)$ encontrada fue de 3 y 4,12 para Leishmania (V.) braziliensis y Leishmania (V.) peruviana respectivamente a diez parásitos/célula; en el tiempo 1, la capacidad infectiva para Leishmania (V.) braziliensis tuvo un incremento de 34,40 veces, el valor alcanzado fue de 102,89 , mientras, en el caso de Leishmania (V.) peruviana la capacidad infectiva alcanzó un valor de 118,68 , que corresponde a un incremento de 28,32 veces; a 15 y 20 parásitos/célula para Leishmania (V.) braziliensis el incremento de la capacidad infectiva fue de 4,2 y 4,9 respectivamente y para Leishmania (V.) peruviana la capacidad infectiva sufrió un incremento de 2,56 y 2,87 a las mismas proporciones (Tabla 1).

Los datos fueron analizados mediante la prueba Wilcoxon $(p<0,05)$ determinándose que no existe diferencia significativa entre ambas especies con relación a la capacidad infectiva de los promastigotes en fase estacionaria. 
Tabla 1. Capacidad infectiva de Leishmania (Viannia) braziliensis y Leishmania (Viannia) peruviana

\begin{tabular}{|c|c|c|c|c|c|c|c|c|c|c|c|c|}
\hline & \multicolumn{6}{|c|}{ Leishmania (Viannia) braziliensis } & \multicolumn{6}{|c|}{ Leishmania (Viannia) peruviana } \\
\hline & \multicolumn{3}{|c|}{ Tiempo 0 (T0) } & \multicolumn{3}{|c|}{ Tiempo 1 (T1) } & \multicolumn{3}{|c|}{ Tiempo 0 (T0) } & \multicolumn{3}{|c|}{ Tiempo 1 (T1) } \\
\hline & $10 \mathrm{p} / \mathrm{cel}$. & $15 \mathrm{p} / \mathrm{cel}$. & 20 p/cel. & $10 \mathrm{p} / \mathrm{cel}$. & $15 \mathrm{p} / \mathrm{cel}$. & 20 p/cel. & $10 \mathrm{p} / \mathrm{cel}$. & 15 p/cel. & $20 \mathrm{p} / \mathrm{cel}$. & $10 \mathrm{p} / \mathrm{cel}$. & $15 \mathrm{p} / \mathrm{cel}$ & $20 \mathrm{p} / \mathrm{cel}$. \\
\hline $\begin{array}{l}\text { Porcentaje de } \varnothing \\
\text { infectados }\end{array}$ & 3 & 16 & 29 & 21,75 & 35 & 48,75 & 4 & 14,75 & 29,75 & 32,5 & 35,5 & 50,75 \\
\hline $\begin{array}{l}\text { Promedio de } \varnothing \\
\text { infectados }\end{array}$ & $6 \pm 1,4$ & $32 \pm 9,9$ & $58 \pm 12,7$ & $43,5 \pm 5$ & $70 \pm 28,3$ & $97,5 \pm 23,3$ & $8 \pm 2,8$ & $29,5 \pm 2,1$ & $59,5 \pm 12,0$ & $65 \pm 7,1$ & $71 \pm 26,9$ & $101,5 \pm 6,4$ \\
\hline $\begin{array}{l}\text { Promedio de } \\
\text { amastigotes por } \varnothing\end{array}$ & 1 & $1,67 \pm 0,4$ & $2,3 \pm 0,1$ & $4,7 \pm 3,4$ & $3,21 \pm 0,4$ & $6,7 \pm 0,3$ & $1,03 \pm 0,2$ & $3,87 \pm 1,7$ & $4,75 \pm 0,1$ & $3,59 \pm 0,2$ & $3,27 \pm 0,6$ & $8 \pm 0,2$ \\
\hline $\begin{array}{l}\text { Capacidad } \\
\text { infectiva }\end{array}$ & 3 & 26,72 & 66,7 & 102,23 & 112,35 & 327,11 & 4,12 & 57,08 & 141,31 & 116,09 & 114,45 & 406 \\
\hline $\begin{array}{l}\text { Incremento con } \\
\text { respecto al T0 }\end{array}$ & - & - & - & 34,08 & 4,2 & 4,9 & - & - & - & 28,32 & 2 & 2,87 \\
\hline
\end{tabular}

Ø: macrófago

p/cel: parásitos/célula

\section{DISCUSIÓN}

El presente estudio permitió evaluar la capacidad infectiva en la línea celular ATCC de macrófago de Canis familiaris DH82, de las formas promastigotes de Leishmania (V.) peruviana y Leishmania (V.) braziliensis, obtenidos de la fase estacionaria; así mismo, se determinó que el mayor porcentaje (13\%) de formas metacíclicas para Leishmania (V.) braziliensis correspondió al día 17,5 posinoculación y para Leishmania (V.) peruviana un porcentaje de 9,5\% en el día 14,5 . El $13 \%$ de formas metacíclicas observado en el presente estudio, es similar al porcentaje (12\%) reportado por Zakai et al., (22) quienes emplearon también el medio Schneider's suplementado al $20 \%$ de SFB, $\mathrm{pH} 7,0^{(22)}$. El número de amastigotes por macrófagos y el número de macrófagos infectados fue determinado mediante la técnica de inmunofluorescencia directa, a

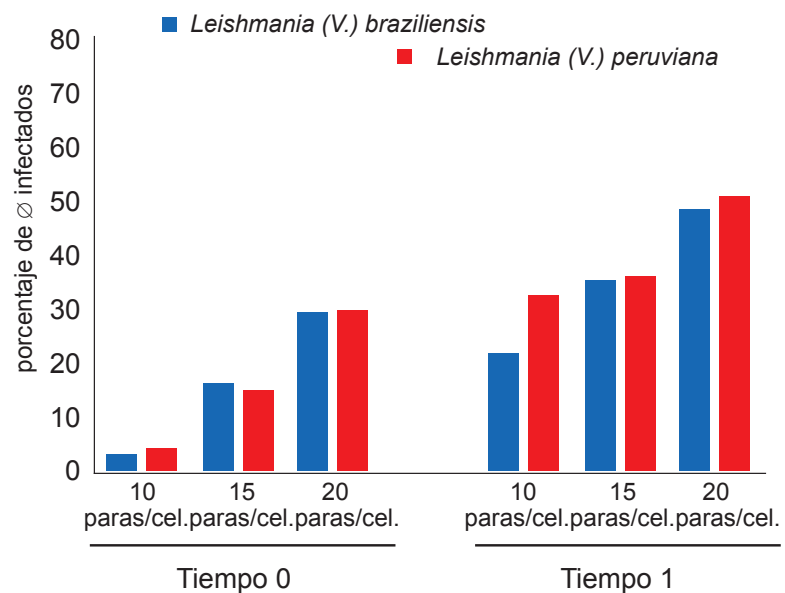

Figura 2. Porcentaje de macrófagos ( $\varnothing)$ infectados con Leishmania (V.) braziliensis y Leishmania (V.) peruviana a diferentes proporciones de parásitos/célula incubadas a $37^{\circ} \mathrm{C}$ con $5 \%$ de $\mathrm{CO}^{2}$ diferencia de trabajos anteriores, donde se empleó la tinción Giemsa ${ }^{(26)}$.

La capacidad infectiva obtenida para el tiempo 0 a 10 parásitos/célula, fue de 3 para Leishmania (V.) braziliensis y para Leishmania (V.) peruviana correspondió a 4,1 , con un porcentaje de macrófagos

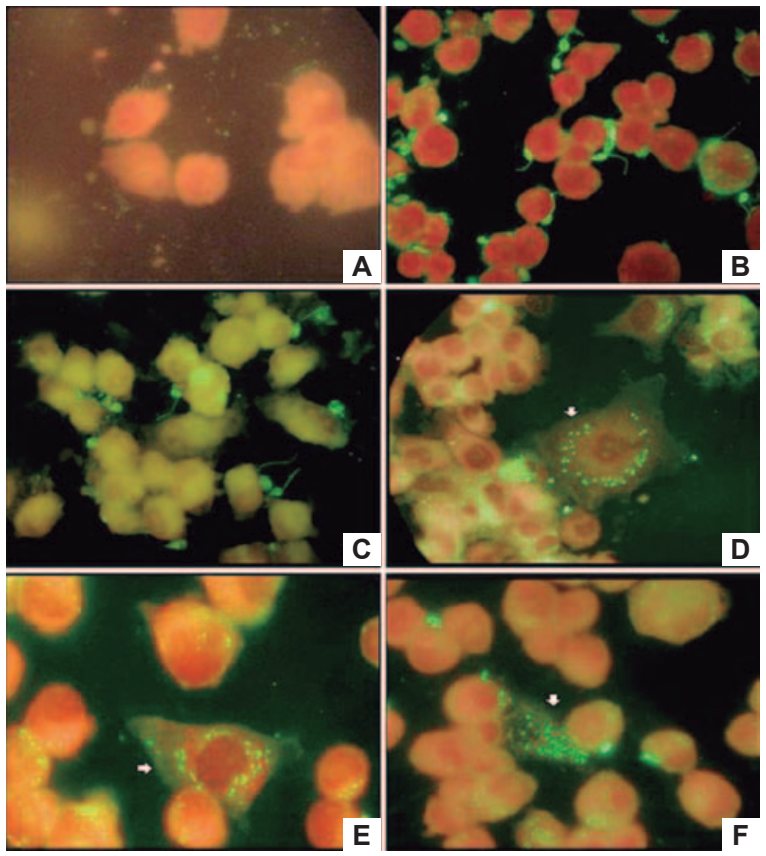

Figura 3. Macrófagos de Canis familiaris ATCC DH82 infectados con Leishmania, visualizado por inmunofluorescencia directa, 100x. El grupo control se muestra en A) macrófagos no infectados; los grupos infección se presentan en B) $T_{0}$ de la infección con Leishmania ( $V$.) peruviana, a 15 parásitos/célula; C) $T_{0}$ de la infección con Leishmania (V.) braziliensis, a 15 parásitos/célula; D, E, F) T de la infección con Leishmania (V.) peruviana a 20 parásitos/célula 
infectados de 3 y $4 \%$ respectivamente, y un amastigote por célula en promedio; mientras que, la capacidad infectiva inicial a 15 y 20 parásitos/célula, en Leishmania (V.) braziliensis alcanzó valores de 26,72 y 66,70 , con un porcentaje de macrófagos infectados de 16 y $29 \%$ y un promedio de 1 a 2 amastigotes por macrófago respectivamente; para Leishmania (V.) peruviana a las mismas proporciones, la capacidad infectiva inicial fue de 57,08 y 141,31 con 14,8 y $29,8 \%$ de macrófagos infectados, con un promedio de 3 y 4 amastigotes por macrófago respectivamente.

La capacidad infectiva de Leishmania (V.) braziliensis a las 48 h posinfección (tiempo 1), a 10 parásitos/célula se incrementó 34 veces y el porcentaje de macrófagos infectados fue de $22 \%$, siete veces mayor en relación al tiempo 0 con un promedio de cuatro amastigotes por macrófago; mientras que, a 15 parásitos/célula, el incremento de la capacidad infectiva fue de 4,2 veces y de 2,2 veces para el porcentaje de macrófagos infectados con respecto al tiempo 0 , con un promedio de tres amastigotes por macrófago; a 20 parásitos/ célula la infectividad se incrementó 4,9 veces, con un $49 \%$ de macrófagos infectados, es decir 1,7 veces en comparación a la capacidad infectiva inicial (tiempo 0), además, presentó un promedio de seis amastigotes por macrófago, similares resultados fueron obtenidos para Leishmania (V.) peruviana (Tabla 1); en ambas especies, la mayor capacidad infectiva o infectividad observada fue a 20 parásitos/célula, al igual que el mayor porcentaje de macrófagos infectados y número de amastigotes por macrófago; sin embargo, el incremento de la capacidad infectiva en relación al tiempo 0 fue mayor a la proporción de diez parásitos/ célula para ambas especies.

Estudios sobre infectividad in vitro de Leishmania (V.) braziliensis realizado por Kweider et al. (27), en macrófagos peritoneales de ratones inoculados con promastigotes obtenidos en medio GLSH (glucosa, lactoalbúmina, suero y hemoglobina), a la proporción de diez parásitos/célula, reportaron que el mayor porcentaje de macrófagos infectados así como el mayor promedio de amastigotes por macrófagos a las $48 \mathrm{~h}$, se obtuvieron con promastigotes en fase estacionaria, en comparación a la fase logarítmica y la fase de declive o muerte, coincidiendo con nuestros resultados; el porcentaje de macrófagos infectados varió entre el $80-90 \%$ con un promedio de cinco a seis amastigotes por macrófago a las $24 \mathrm{~h}$ y entre $90-100 \%$ con promedio de seis a siete amastigotes por macrófago a las 48 horas ${ }^{(27)}$, a diferencia del presente estudio donde los valores encontrados fueron menores.
Del análisis realizado en el presente estudio, se determinó que no existe diferencia significativa entre las especies a un nivel de confianza de 0,95; en el tiempo 0, para 10,15, y 20 parásitos/célula, se encontró diferencia significativa entre las tres proporciones; mientras que, al tiempo 1, la diferencia significativa estuvo entre 10 y 20 parásitos/célula (datos no mostrados); nuestros resultados difieren de lo obtenido in vitro por Gamboa et al (28), quienes empleando macrófagos de murinos RAW 264, inoculados con 30 parásitos/célula de formas metacíclicas obtenidas a $\mathrm{pH} 5,5$, en gradiente de ficoll a 30 parásitos/célula, quienes encontraron que el porcentaje de macrófagos infectados y el número de amastigotes/célula fue mayor para Leishmania (V.) braziliensis especialmente la cepa de origen mucocutánea (MHOM/PE/91/LC2043) seguido por la cepa de origen cutáneo (MHOM/PE/91/ LC2177); las especies de Leishmania (V.) peruviana mostraron una menor infectividad principalmente la cepa procedente de la sierra sur del Perú (MHOM/ PE/90/LCA08) en comparación a la cepa procedente de la sierra norte (MHOM/PE/90/HB86) ${ }^{(28)}$

La diferencia con los resultados del presente estudio, podría estar relacionada con la interacción de factores como la forma parasitaria empleada (formas metacíclicas puras), la población parasitaria (homogénea o heterogénea), la línea celular empleada, donde la susceptibilidad está ligada a su naturaleza, siendo los cultivos primarios los que presentan mayor susceptibilidad, no obstante, presentan tiempo limitado de crecimiento y bajo rendimiento ${ }^{(29)}$, además, las diferentes líneas celulares secundarias presentan receptores para los diversos factores de virulencia de la especie de Leishmania ${ }^{(28)}$; otros factores serían el número de pasajes ${ }^{(18,30)}$, y el $\mathrm{pH}$ del medio de cultivo empleado en la obtención de las formas virulentas o metaciclícas ${ }^{(22,28,30)}$.

Basados en estos resultados, se recomienda evaluar la capacidad infectiva de los promastigotes metacíclicos de cepas de Leishmania (V.) peruviana y Leishmania (V.) braziliensis en líneas celulares, empleando parásitos recientemente aislados y promastigotes metacíclicos obtenidos in vitro; a fin de determinar el modelo de infección in vitro más adecuado, que permita efectuar estudios de susceptibilidad a las drogas leishmanicidas existentes y a otras alternativas terapéuticas, para el control de la enfermedad.

Contribuciones de autoría: KDCS y NMRP han participado en la concepción del artículo, la recolección de datos, su redacción del artículo y además NMRP brindó asesoría técnica, ACSJ y ACT participaron en el aporte de material de estudio, COC participó con la asesoría estadística y GSMG 
participó en la revisión crítica del artículo y aprobación de su versión final.

Fuentes de financiamiento: el presente trabajo conto con el apoyo de insumos y materiales, además del uso de los equipos del Laboratorio de Leishmaniasis del Centro Nacional de Salud Pública del Instituto Nacional de Salud, Perú.

Conflictos de interés: los autores declaran no tener conflictos de interés en la publicación del artículo.

\section{REFERENCIAS BIBLIOGRÁFICAS}

1. Sanchez-Saldaña L, Saenz-Anduaga E, Pancorbo-Mendoza J, Zegarra-DelCarpio R, Garces-Velasco N, RegisRoggero A. Leishmaniasis. Dermatología Peruana. 2004;14(2):82-98.

2. Alvar J, Vélez ID, Bern C, Herrero $\mathrm{M}$, Desjeux $\mathrm{P}$, Cano $\mathrm{J}$, et al. Leishmaniasis worldwide and global estimates of its incidence. PLoS One. 2012;7(5):e35671. doi: 10.1371/ journal.pone.0035671.

3. Red Nacional de Epidemiología (RENACE), Dirección General de Epidemiología. Sala situacional de salud Perú. Semana Epidemiológica 53 - 2014. Lima: MINSA; 2014.

4. Chang K, Reed SG, Mcgwire BS Soong L. Leishmania model for microbial virulence: the relevance of parasite multiplication and pathoantigenicity. Acta Trop. 2003 Mar;85(3):375-90.

5. Rivas L, Moreno J, Cañavate C, Alvar J. Virulence and disease in leishmaniasis: what is relevant for the patient?. Trends Parasitol. 2004 Jul;20(7):297-301.

6. Chang KP, Chaudhuri G, Fong D. Molecular determinants of Leishmania virulence. Annu Rev Microbiol. 1990;44:499-529.

7. Handman E, Bullen DV. Interaction of Leishmania with the host macrophage. Trends Parasitol. 2002 Aug;18(8):332-4.

8. Contreras I, Gómez MA, Nguyen O, Shio MT, McMaster RW, Olivier M. Leishmania-Induced Inactivation of the Macrophage Transcription Factor AP-1 Is Mediated by the Parasite Metalloprotease GP63. PLoS Pathog. 2010 Oct 14;6(10):e1001148. doi: 10.1371/ journal.ppat.1001148.

9. Shapiro-Ilan DI, Fuxa JR, Lacey LA, Onstad DW, Kaya HK. Definitions of pathogenicity and virulence in invertebrate pathology. J Invertebr Pathol. 2005 Jan;88(1):1-7.
10. Thomas SR, Elkinton JS. Pathogenicity and virulence. J Invertebr Pathol. 2004 Mar;85(3):146-51.

11. Casadevall A, Pirofski L. Hostpathogen interactions: the attributes of virulence. J Infect Dis. 2001 Aug 1;184(3):337-44.

12. Casadevall A, Pirofski LA. Hostpathogen interactions: redefining the basic concepts of virulence and pathogenicity. Infect Immun. 1999 Aug;67(8):3703-13.

13. Muskus CE, Villa MM. Metaciclogénesis: un proceso fundamental en la biología de Leishmania. Biomédica. 2002;22(2):167-77.

14. Handman E. Cell biology of Leishmania. Adv Parasitol. 1999;44:1-39.

15. Sereno D, Cordeiro-da-Silva A, Mathieu-Daude F, Ouaissi A. Advances and perspectives in Leishmania cell based drug-screening procedures. Parasitol Int. 2007 Mar;56(1):3-7.

16. Stinson S, Sommer JR, Blum JJ. Morphology of Leishmania braziliensis: changes during reversible heat-induced transformation from promastigote an ellipsoidal form. J Parasitol. 1989 Jun;75(3):431-40.

17. Almeida MC, Cuba CA, de Sa CM, Pharoah MM, Howard KM, Miles MA. Metacyclogenesis of Leishmania (Viannia) braziliensis in vitro: evidence that lentil lectin is a marker of complement resistance and enhanced infectivity. Trans R Soc Trop Med Hyg. 1993 May-Jun;87(3):325-9.

18. Da Silva R, Sacks DL. Metacyclogenesis is a major determinant of Leishmania promastigote virulence and attenuation. Infect Immun. 1987 Nov;55(11):2802-6.

19. Soares RPP, Cardoso TL, Barron T, Araújo MS, Pimenta PFPP, Turco SJ. Leishmania braziliensis: a novel mechanism in the lipophosphoglycan regulation during metacyclogenesis. Int J Parasitol. 2005 Mar;35(3):245-53.

20. De Assis RR, Ibraim IC, Nogueira PM, Soares RP, Turco SJ. Glycoconjugates in New World species of Leishmania: polymorphisms in lipophosphoglycan and glycoinositolphospholipids and interaction with hosts. Biochim Biophys Acta. 2012 Sep;1820(9):1354-65. doi: 10.1016/j.bbagen.2011.11.001.

21. Serafim TD, Figueiredo AB, Costa PA, Marques-da-Silva EA, Gonçalves $\mathrm{R}$, de Moura SA, et al. Leishmania metacyclogenesis is promoted in the absence of purines. PLoS Negl Trop Dis. 2012;6(9):e1833. doi: 10.1371/ journal.pntd.0001833.

22. Zakai HA, Chance ML, Bates PA. In vitro stimulation of metacyclogenesis in Leishmania braziliensis, L. donovani, L. major and L. mexicana. Parasitology. 1998 Apr;116(Pt4):305-9.

23. Ibraim IC, de Assis RR, Pessoa NL, Campos MA, Melo MN, Turco SJ, et al. Two biochemically distinct lipophosphoglycans from Leishmania braziliensis and Leishmania infantum trigger different innate immune responses in murine macrophages. Parasit Vectors. 2013 Mar 7;6:54. doi: 10.1186/1756-3305-6-54.

24. NievesE,Rondón M.Metaciclogénesisy desarrollo de Leishmania (Leishmania) amazonensis (Kinetoplastida: Trypanosomatidae) en Lutzomyia ovallesi (Diptera: Psychodidae). Revista de la Sociedad Venezolana de Microbiología. 2010;30:121-7.

25. Monzote Hidalgo L, Montalvo Alvarez AM, Fonseca Géigel L, Pérez Pineiro R, Suarez Navarro M, Rodriguez Cabrera H. Efecto de derivados de la tiadiazina sobre la capacidad infectiva de promastigotes de Leishmania amazonensis. Rev Cuba Med Trop. 2006;58(1):25-9.

26. Gamboa Marín YA, Delgado Murcia LG. Estudio de la infección de 
Leishmanias del complejo Viannia mediante citometría de flujo y coloración de Giemsa empleando líneas de macrófagos humanos y murinos (U937 y J-774). Rev Colomb Cienc Quím Farm. 2008;37(1):84-95.

27. Kweider M, Lemesre JL, Darcy F, Kusnierz JP, Capron A, Santoro F. Infectivity of Leishmania braziliensis promastigotes is dependent on the increasing expression of a 65,000-dalton surface antigen. J Immunol. 1987 Jan;138(1):299-305.
28. Gamboa D, Torres K, De Doncker S, Zimic M, Arévalo J, Dujardin JC. Evaluation of an in vitro and in vivo model for experimental infection with Leishmania (Viannia) braziliensis and L . (V.) peruviana. Parasitology. 2008 Mar;135(3):319-26.

29. Meza C, Muñoz DL, Echeverry MCM, Vélez ID, Robledo SM, Mesa CV, et al. Susceptibilidad in vitro a infección por difiere según tipo de macrófagos. Salud UIS. 2010;42(61):200-11.
30. López López MC. Parasitología molecular. Madrid: CSIC-CSIC; 1993.

Correspondencia: Gloria Minaya-Gómez Dirección: Capac Yupanqui 1400 Jesús María. Lima, Perú

Telefono: (01)748 1111 anexo 2167

Correo electrónico:gminaya22@gmail.com

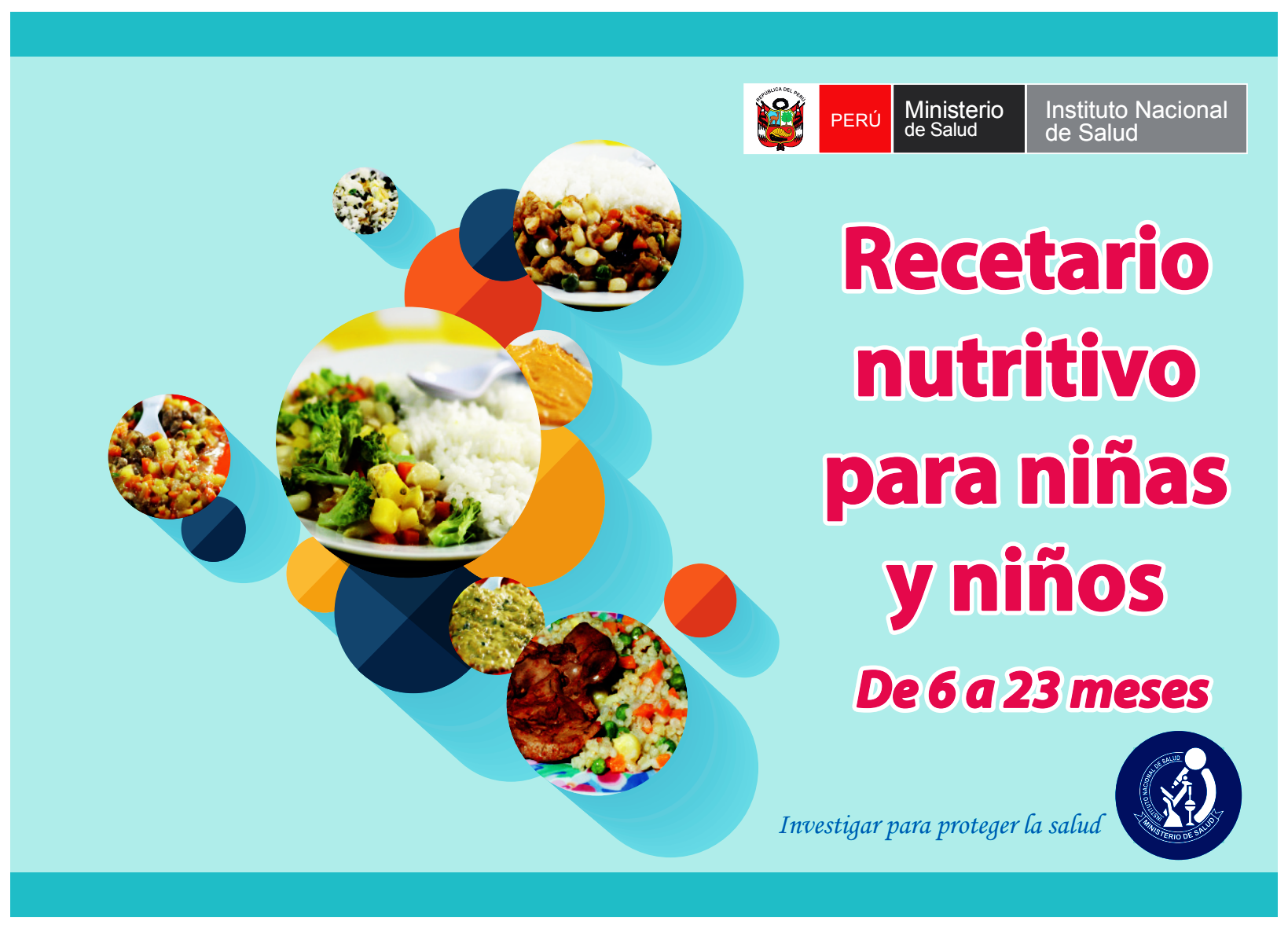

